\title{
Actividad antiplasmodial in vitro de metabolitos secundarios de Solanum nudum provenientes de dos regiones de Colombia
}

\author{
EnA PATRICIA López-BARRIOS ${ }^{1}$ \\ ADRIANA LUCÍA PABÓN-VIDAL ${ }^{1}$ \\ Silvia Blair-TRUJILlo ${ }^{1}$ \\ Paula ANDrea Morales-Morales ${ }^{2}$ \\ CARlos Alberto PeláEz-JARAMillo 3 \\ Pedronel Araque-Marín ${ }^{4}$
}

\section{Resumen}

Solanum nudum es una planta nativa de Colombia, reconocida y usada para el tratamiento de la fiebre asociada a la malaria, específicamente en el área del pacífico del departamento de Nariño. En la presente investigación se realizó un estudio fitoquímico comparativo de la producción de los compuestos esteroidales de $S$. nudum provenientes de dos regiones de Colombia y su respectiva actividad antiplasmodial. La colección de las hojas de $S$. nudum se realizó en los municipios de Cocorná (Antioquia) y Tumaco (Nariño) en los meses de noviembre 2010 y junio 2011. La extracción de los metabolitos esteroidales presentes en las hojas colectadas de S. nudum se efectuó con solventes de baja, media y alta polaridad. El contenido de los metabolitos esteroidales se determinó por cromatografía líquida de alta resolución en fase reversa. La actividad antiplasmodial de los extractos fue evaluada en la cepa 3D7 de Plasmodium falciparum y su posible citoxicidad en la línea celular HepG-2. El contenido de compuestos esteroidales y la actividad antiplasmodial fue superior para los extractos de las regiones y los meses donde la precipitación promedio del lugar fue alterada debido al fenómeno de La Niña. Los extractos con mayor efecto antiplasmodial y citotóxico citotoxicidad fueron los obtenidos en el municipio de Tumaco.

1 Grupo de Malaria, Universidad de Antioquia, Medellín, Colombia.

2 Grupo Herbario, Universidad de Antioquia, Medellín-Colombia.

3 Grupo Interdisciplinario de Estudios Moleculares-GIEM, Universidad de Antioquia, Medellín, Colombia.

4 Grupo de Investigación e Innovación en Formulaciones Químicas, Universidad EIA, Envigado, Colombia.

Autor de correspondencia: Araque Marín (Pedronel): Universidad EIA, Sede de Las Palmas: Km 2 + 200 Vía al Aeropuerto José María Córdova, Envigado, Colombia. C.P.: 055428. Teléfono: (574)3549090 Opción 1, Ext. 234. Correo electrónico: pedronel.araque@eia.edu.co
Historia del artículo:

Artículo recibido: 29-X-2016 / Aprobado: 24-V-2018

Disponible online: 3 de agosto 2018

Discusión abierta hasta abril de 2020 
Palabras claves: Solanum nudum; malaria; Plasmodium falciparum; metabolitos esteroidales; Cocorná; Tumaco.

\section{Antiplasmodial activity in vitro of secondary metabolites of Solanum nudum from two regions of Colombia}

\section{Abstract}

Solanum nudum is a plant native to Colombia, is recognized and is used for the treatment of fever associated with malaria, specifically in the Pacífico area of Nariño department. In this research a comparative study of phytochemical production of steroidal compounds of S. nudum from two regions of Colombia and their respective antiplasmodial activity was carried out. The collection of the leaves of $S$. nudum was conducted in the municipalities of Cocorná (Antioquia) and Tumaco (Nariño) in the months of November 2010 and June 2011. The extraction of steroid metabolites present in the leaves were collected from $S$. nudum was performed with solvents low, medium and high polarity. The content of steroidal metabolites was determined by high resolution liquid chromatography in reverse phase. The antiplasmodial activity of the extracts was evaluated in 3D7 strain of Plasmodium falciparum and its potential cytotoxicity on the cell line HepG-2. The content of steroidal compounds and antiplasmodial activity was higher on extracts from regions and months where the mean precipitation was influenced due to La Niña phenomenon. The extracts more antiplasmodial effect and cytotoxicity were obtained from Tumaco municipality.

Keywords: Solanum nudum; malaria; Plasmodium falciparum; steroidal metabolites; Cocorná; Tumaco.

\section{Actividade in vitro antiplasmódica de metabolitos secundários de Solanum nudum a partir de duas regiões da Colômbia}

\section{Resumo}

Solanum nudum é uma planta nativa da Colômbia, é reconhecido e é usado para o tratamento da febre associada à malária, especificamente na área pacífica do departamento de Nariño. Nesta pesquisa um estudo comparativo da produção fitoquímico de compostos esteróides de $S$. nudum de duas regiões da Colômbia e sua respectiva actividade antiplasmódica foi realizado. A coleta das folhas de $S$. nudum foi realizado nos municípios de Cocorná (Antioquia) e Tumaco (Nariño), nos meses de novembro de 2010 e junho de 2011. A extração de metabólitos esteróides presentes nas folhas foram coletadas de $S$. nudum foi realizada com solventes de baixa, média e alta polaridade. 0 teor de metabolitos esteróides foi determinada por cromatografia líquida de alta resolução em fase reversa. A actividade antiplasmódica dos extractos foi avaliada em 3D7 estirpe de Plasmodium falciparum e a sua citotoxicidade potencial na linha de células HepG-2. 0 teor de compostos esteróides e atividade antiplasmódica foi maior para os extratos regiões e meses em que a precipitação média do site foi alterada devido ao fenômeno do La Niña. Os extratos mais efeito antiplasmódica e citotoxicidade foram obtidos no município de Tumaco.

Palavras-chave: Solanum nudum; malária; Plasmodium falciparum; metabólitos esteroides; Cocorná; Tumaco. 


\section{Introducción}

Solanum nudum es un arbusto que se distribuye desde México hasta Paraguay incluyendo las islas del Caribe y se encuentra desde cero hasta 2500 msnm. En Colombia se encuentra en 16 de los 32 departamentos, habitando principalmente en áreas abiertas o intervenidas como: caminos, carreteras y rastrojos (Orozco, et al. 2015). Es una planta reconocida y usada para el tratamiento de la fiebre asociada a la malaria por las comunidades humanas de la costa pacífica donde es localmente conocida como "zapata" o "zapatico", especialmente en el departamento de Nariño donde el uso de esta planta por médicos tradicionales del municipio de Tumaco ha despertado el interés por estudiar su composición química y actividad biológica (Blair y Madrigal, 2005).

De la planta se han aislado compuestos esteroidales llamados Tumacona A (SN-1), Tumacona B (SN-2), Tumacosido A (SN-3), Tumacosido B (SN-5), Tumaquenona (SN-4) y la Diosgenona (Saez, et al. 1998). Estos metabolitos mostraron actividad antiplasmodial in vitro sobre Plasmodium falciparum (Pabón, et al. 2002). Así mismo, se ha reportado que los esteroides aislados de $S$. nudum no presentan actividad mutagénica (Pabón, et al. 2003), clastogénica (Álvarez, et al. 2004) ni tóxica (Londoño, et al. 2006). Además, extractos acuosos de $S$. nudum presentan actividad antimalárica in vivo en ratones infectados con Plasmodium berghei (Echeverri, et al. 2001).

Otros estudios indican que los esteroides de S. nudum disminuyen el desarrollo esporogónicos de Plasmodium vivax en Anopheles albimanus y en células hepáticas HepG2 (Londoño, et al. 2006). Sin embargo, se ha establecido que el tiempo entre la colecta, el almacenamiento y el procesamiento del material afectan la composición química y acción biológica de la planta (López, et al. 2011), infiriendo las diferencias en la actividad antiplasmodial podrían estar relacionadas con la degradación biológica de la planta (Stafford, et al. 2005; Christos, et al. 2010).
Diferencias en los aspectos ecológicos de poblaciones distantes de $S$. nudum (producción de frutos, diversidad de visitantes florales, herbívoros y parásitos asociados), así como diferencias en su hábitat (composición del suelo, temperatura, humedad relativa y luminosidad), pueden afectar la actividad biológica (Morales, 2011), dado que factores ambientales como la altitud, temperatura, humedad y el tipo de suelo, generan alteraciones en las rutas bioquímicas de las plantas, produciendo un incremento y/o disminución de metabolitos como ácidos grasos, compuestos volátiles, entre otros (Boira y Blanquer, 1998). Un ejemplo de este efecto es los estudios sobre la composición química del romero (Rosmarinus officinalis) en el cual la localidad como la fecha de colecta (Celiktas, et al. 2007) afectan la producción de aceites esenciales y su correspondiente actividad antimicrobiana.

En la presente investigación se realizó un estudio fitoquímico comparativo de la producción de los compuestos esteroidales (SN-1, SN-2, SN-3, SN4 , SN-5 y Diosgenona) de la planta $S$. nudum provenientes de dos regiones diferentes de Colombia y su respectiva actividad antiplasmodial in vitro.

\section{Materiales y métodos}

\section{Reactivos}

Los solventes: hexano, diclorometano, metanol y dimetilsulfoxido (DMSO) de Pueza $\geq 99 \%$ fueron obtenidos de Merck. Los medios RPMI1640 (Roswell Park Memorial Institute), DMEM (Dulbecco's Modified Eagle Medium), SFB (Suero Fetal Bovino) y el reactivo MTT [bromuro 3-(4,5-dimetiltiazol-2-ilo)-2,5-difeniltetrazolio)] fueron obtenidos de Sigma-Aldrich Chemical Co. Ltd., Proanalysis.

\section{Recolección del material vegetal}

Con el fin de disminuir el efecto de los tiempos de procesamiento del material vegetal en los análisis, se colectó $S$. nudum en dos localidades distantes de Colombia, de forma que el tiempo transcurrido entre en la colecta y procesamiento fuera el mismo. 
La colección de las hojas de $S$. nudum se realizó en dos localidades con condiciones climáticas y fisiográficas diferentes y en dos épocas del año. Los lugares de colecta fueron en la vereda la Piñuela, municipio de Cocorná (Departamento de Antioquia, región Andina, 600'18” N; 7508'16” W, 1150 m de altitud) y la vereda Inguapí el Guadual, municipio de Tumaco (Departamento de Nariño, en la región Pacífica $1^{\circ} 41^{\prime} 03^{\prime \prime} \mathrm{N}$; 7847' 01”'W, 12 m altitud). La colecta del material vegetal se realizó en noviembre 2010 y junio 2011 en ambos lugares. De cada localidad fue colectado un Voucher, los cuales se encuentran depositados en el Herbario de la Universidad de Antioquia (HUA) bajo los registros de herbario $\mathrm{N}^{\circ} 179201$ y 179202.

\section{Obtención de extractos de S. nudum}

El material vegetal se secó al ambiente y a la sombra (aproximdadmente a $22{ }^{\circ} \mathrm{C}$ ), durante 10 días y se molió hasta tener un tamaño de partícula de $5 \mathrm{~mm}$. Se tomaron 20 gramos del material vegetal seco y molido y se sometieron a extracción sucesiva por el método Soxhlet durante cinco horas, empleando $250 \mathrm{~mL}$ de solvente de baja, media y alta polaridad: hexano $\left(0,08\right.$ Debye a $\left.20^{\circ} \mathrm{C}\right)$, diclorometano $\left(1,14\right.$ Debye a $\left.20^{\circ} \mathrm{C}\right)$ y metanol $(1,69$ Debye a $20^{\circ} \mathrm{C}$ ). Posteriormente los extractos fueron concentrados a presión reducida por rotaevaporación.

\section{Cuantificación de esteroides en ex-} tractos de $S$, nudum

La cuantificación de los metabolitos esteroidales presentes en las hojas colectadas de $S$. nudum se realizó por cromatografía liquida de alta resolución (HPLC) en fase reversa, utilizando un cromatógrafo líquido Agilent Technologies 1200 Series (Agilent Technologies, Wilmington, USA), con degasificador G1322A, bomba cuaternaria G1311A, inyector automático G1329A y detector de arreglo de diodos (DAD) G1315DHPLC. Las condiciones cromatográficas fueron: columna LiChroCART® 250-4 cartridges LiChrospher® 100 RP-18 (5 $\mu \mathrm{m})$, a una temperatura de columna de $25^{\circ} \mathrm{C}$, volumen de inyección de $20 \mu \mathrm{L}$, longitud de onda de $254 \mathrm{~nm}$, flujo de $1,0 \mathrm{ml}^{\mathrm{min}} \mathrm{mi}^{-1} \mathrm{y}$ como fase móvil acetonitrilo/agua por programación de gradientes. Los extractos fueron filtrados por medio de filtro de nylon de 0,45 um (López, et al. 2014). Como compuestos patrones se usaron los metabolitos esteroidales de S. nudum almacenados en el Laboratorio del Grupo Malaria con previa caracterización molecular mediante un análisis espectroscópico de resonancia magnética nuclear, en el que se compararon las señales presentes en los espectros con los datos reportados en la literatura por Saez et al. (1999). Se utilizó un espectrómetro Bruker, $300 \mathrm{MHz}$ para $1 \mathrm{H}$ y $75 \mathrm{MHz}$ para 13C, en 1D (RMN- 13C, RMN-1H), utilizando como solvente cloroformo deuterado $\left(\mathrm{CDCl}_{3}\right)$.

TABLA 1. CONDICIONES AMBIENTALES Y ECOLÓGICAS DE LAS LOCALIDADES DONDE FUE COLECTADA LA PLANTA S.
nudum
\begin{tabular}{l|c|c}
\multicolumn{1}{|c|}{ Parámetros ambientales } & Tumaco & Cocorná \\
\hline Luminosidad & $15392,8 \pm 0,1$ lux & $25299,8 \pm 0,1$ lux \\
\hline Temperatura ambiente & $31,04 \pm 0,05^{\circ} \mathrm{C}$ & $29,28 \pm 0,05^{\circ} \mathrm{C}$ \\
\hline Temperatura del suelo & $19,42 \pm 0,05 \mathrm{C}$ & $15,68 \pm 0,05^{\circ} \mathrm{C}$ \\
\hline Humedad relativa & $56,89 \pm 0,02 \%$ & $51,94 \pm 0,02 \%$ \\
\hline Carbono presente en el suelo & $1,28 \pm 0,01 \%$ & $3,26 \pm 0,01 \%$ \\
\hline Insectos herbívoros & 49 especies & 44 especies \\
\hline Insectos parásitos permanentes & 24 especies & 17 especies \\
\hline Otros insectos (huéspedes temporales) & 90 especies & 69 especies \\
\hline Visitantes florales & 3 especies & 17 especies \\
\hline Cantidad de frutos & $238,5 \pm 0,1$ frutos $/$ mes & $611,2 \pm 0,1$ frutos $/$ mes \\
\hline
\end{tabular}


El tiempo de corrido fue de cuarenta minutos para los cuales la fase móvil acetonitrilo/agua fue programada para los siguientes gradientes: primeros diez minutos de acetonitrilo/agua en relación 35:65, continuando cinco minutos con cambio de gradiente acetonitrilo/agua de 35:65 a 80:20, para este gradiente eluyeron los compuestos SN-4, SN-5 y SN-3 con tiempos de retención de 12,317; 14,145 y 14,850 minutos respectivamente. Luego siete minutos de acetonitrilo/agua en relación 80:20, en este gradiente eluyeron los compuestos $\mathrm{SN}-2$ y SN-1 con tiempos de retención de 16,568 y 17,657 minutos. Posteriormente trece minutos con cambio de gradiente acetonitrilo/agua de 80:20 a 90:10 y finalmente cinco minutos de acetonitrilo/agua en relación 90:10, donde eluyó la Diosgenona con un tiempo de retención de 37,492 minutos. Por medio de la aplicación de estos gradientes se pueden clasificar los patrones de esteroides en tres grupos: polaridad alta (SN-4, SN-5 y SN-3) polaridad media (SN-2 y SN-1) y polaridad baja (Diosgenona).

Para la construcción de la curva de calibración, se prepararon seis niveles de concentraciones desde $0,5 \mathrm{ug} / \mathrm{ml}$ - 160, para los compuestos $\mu \mathrm{g} / \mathrm{mL}$ para los compuestos SN-1, SN-2, SN-3 y SN-5 y para $\mathrm{SN}-4$ y diosgenona las concentraciones evaluadas fueron: 3,0, y $160 \mu \mathrm{g} / \mathrm{mL}$. Cada concentración se preparó a partir de una solución madre de 1000 g/ $\mathrm{mL}$ de cada compuesto.

\section{Evaluación de la actividad antiplas- modial in vitro}

De cada extracto se prepararon soluciones concentradas para la evaluación de la actividad antiplasmodial de $10 \mathrm{mg} / \mathrm{mL}$ en dimetilsulfóxido (DMSO). A partir de esta solución se tomaron $50 \mu \mathrm{L}$ y se ajustaron hasta $1000 \mu \mathrm{L}$ con medio RPMI-1640 completo sin hipoxantina, obteniendo una concentración final de $0,5 \mathrm{mg} / \mathrm{mL}$.

La actividad antiplasmodial in vitro de los extractos obtenidos de la planta $S$. nudum se realizaron en placas de 96 pozos de fondo plano marca Falcon®. Se evaluaron 7 concentraciones de cada extracto $(100 ; 50 ; 25 ; 12,5 ; 6,25 ; 3,125$ y $1,56 \mu \mathrm{g} /$ $\mathrm{mL}$ ), preparadas a partir de diluciones seriadas de la solución concentrada de $0,5 \mathrm{mg} / \mathrm{mL}$. Cada concentración fue evaluada por duplicado (Bravo, et al. 1999; Desjardins, et al. 1979).

La actividad c antiplasmodial se evaluó en la cepa 3D7 de P. falciparum, la cual es un clon obtenido a partir de un aislado de paciente en Holanda. Esta cepa es sensible a cloroquina, pirimetamina, cicloguanil y atovacuona; pero es resistente a sulfadoxina (Walliker et al. 1987). Para llevar a cabo los experimentos se preparó una una suspensión de glóbulos rojos parasitados con un hematocrito del $2 \%$, parasitemia del $1 \%$ e hipoxantina tritiada por pozo de $0,8 \mu \mathrm{Ci}$.mL ${ }^{-1}$ siguiendo la metodología descrita por Desjardins, R et al. 1979

El cultivo con los tratamientos de los diferentes extractos se incubó a $37{ }^{\circ} \mathrm{C}$ durante 48 horas en atmósfera de $5 \% \mathrm{CO}_{2}, 5 \% \mathrm{O}_{2}$ y $90 \%$ de $\mathrm{N}_{2}$. El plato se llevó a $-20{ }^{\circ} \mathrm{C}$ y posteriormente se descongeló para provocar la hemólisis de los eritrocitos, lo cual permite la liberación de los ácidos nucleícos del parásito marcados con hipoxantina tritiada. Estas muestras fueron depositadas en un papel filtro de fibra de vidrio diseñado para contener muestras biológicas (PerkinElmer ${ }^{\circledR}$ ) con ayuda de un colector semi-automático (PerkinElmer ${ }^{\circledR}$ ) y la reactividad, la cual es dependiente de la parasitemia en cada tratamiento fue leídos en un contador de centelleo beta Perkin Elmer, 2450 microplate counter. La lectura se expresó en cpm (cuentas por minuto). Los porcentajes de parasitemia se analizaron mediante una regresión no lineal dependiendo de la pendiente, con el programa GraphPad Prism $^{\text {TM }}$ versión 5.01, para hallar la concentración que inhibe el $50 \%$ del crecimiento parasitario $\left(\mathrm{IC}_{50}\right)$.

\section{Evaluación de la actividad citotóxica}

La evaluación de la toxicidad de los extractos obtenidos de la planta $S$. nudum, se determinó mediante la medición de la viabilidad y proliferación in vitro de la línea celular HepG-2, como modelo para evaluar el potencial efecto tóxico sobre los 
hepatocitos, que constituyen la primera célula hospedera para el $P$. falciparum en el humano y la única célula que invade el parásito en este hospedero con capacidad de dividirse.

Para cada ensayo se preparó una solución patrón de cada extracto a una concentración de 20 $\mathrm{mg} / \mathrm{mL}$ en DMSO al 96\% (2 mg de cada extracto en $100 \mu \mathrm{L}$ de DMSO). En medio DMEM F-12 completo (10\% de SFB), se prepararon siete diluciones seriadas a partir de la solución patrón.

En cámara de Neubauer se contaron las células HepG-2 y se sembraron en una placa de 96 pozos de fondo plano $2 \times 10^{5}$ células/mL en $100 \mu \mathrm{L}$ de medio DMEM-F-12 suplementado con SFB al $10 \%$ y se incubaron a $37^{\circ} \mathrm{C}$ durante 24 horas en atmosfera de $5 \%$ de $\mathrm{CO}_{2}$ para permitir la formación de la monocapa. Luego las células fueron lavadas con solución salina al $0,85 \%$ y se agregaron $100 \mu \mathrm{L}$ de cada una de las cuatro concentraciones de los extractos. Cada concentración del extracto y los controles se evaluaron por triplicado en 2 ensayos. Posteriormente, las placas se incubaron a $37^{\circ} \mathrm{C}$ durante 48 horas, se eliminó el medio de cultivo y las células se lavaron con solución salina a $0,85 \%$, se agregaron $30 \mu \mathrm{L}$ de MTT ((3-(4,5-dimethylthiazol-2-yl)-2,5-diphenyltetrazolium bromide) a una concentración de $2 \mathrm{mg} /$ $\mathrm{mL}$ preparado en medio de cultivo y las células se incubaron nuevamente por 4 horas. Después de la incubación, se agregaron $130 \mu \mathrm{L}$ de DMSO al 96\%. La lectura de la viabilidad celular se realizó mediante la medición de la producción de formazán (producido a partir del metabolismo del MTT), la cual se midió en un espectofotómetro Multiskan Spectrum Thermo scientific medido a $570 \mathrm{~nm}$ de exitación y
595 nm de emisión (Mosmann, 1983). Las densidades ópticas obtenidas se analizaron con el programa GraphPad Prism 5 para hallar la concentración tóxica para el $50 \%$ de las células de prueba $\left(\mathrm{TC}_{50}\right)$.

\section{Análisis estadístico}

Para comparar las concentraciones de los compuestos en cada uno de los extractos y entre las diferentes fechas de colecta se empleó la prueba ANOVA. Cuando se encontraron diferencias estadísticamente significativas se aplicaron los test de comparaciones múltiples por parejas de compuestos; se usó el test de Tukey cuando las varianzas fueron iguales y el Test de Games Howell cuando las varianzas fueron diferentes. Para comparar las concentraciones de los compuestos y las actividades biológicas de los extractos entre los dos municipios se usó la prueba T de Student para muestras independientes. En todos los casos se usó un valor p de significancia menor a 0,05. Estas pruebas se hicieron con el programa estadístico SPSS versión 19 y para las curvas de calibración se usó el software STATGRAPHICS Centurión 5,0.

\section{Resultados y Discusión}

\section{Recolección del material vegetal}

En la Tabla 2 se presentan los datos geográficos de los lugares de colecta, las cuales fueron realizadas en la vereda la piñuela del municipio de Cocorná y la vereda Inguapí el Guadal del municipio de Tumaco. Nótese que ambas localidades también difieren en varios aspectos ecológicos y microclimáticos, descritos previamente en la Tabla 1.

TABLA 2. PARÁMETROS DE COLECTA DE LAS HOJAS DE S. nudum

\begin{tabular}{c|c|c|c|c|} 
Colecta & Municipio & Vereda & Coordenadas & msnm \\
\cline { 1 - 2 } Nov-2010 & Cocorná & La piñuela & $6^{\circ} 00^{\prime} 18^{\prime \prime} \mathrm{N} ; 75^{\circ} 08^{\prime} 16^{\prime \prime} \mathrm{W}$ & 1150 \\
\cline { 1 - 2 } & Tumaco & Inguapí el Guadual & $1^{\circ} 41^{\prime} 13^{\prime \prime} \mathrm{N} ; 78^{\circ} 46^{\prime} 43^{\prime \prime} \mathrm{W}$ & 12 \\
\cline { 1 - 2 } & & & &
\end{tabular}




\section{Extractos de $S$, nudum}

El porcentaje promedio en masa de los extractos totales obtenidos de la planta $S$. nudum fue de $33 \pm 5 \%$, para el cual el 1,7 $\pm 0,5 \%$ corresponde la extracción realizada con hexano; 5,2 \pm 0,6\% a la realizada con diclorometano y $26 \pm 5 \%$ a la realizada con metanol. En la Tabla 3 se expresan los porcentajes de extracto de la planta de $S$. nudum proveniente de los municipios de Cocorná y Tumaco.

\section{Cuantificación de esteroides en ex-} tractos de $S$, nudum

Los análisis estadísticos de los patrones esteroidales (SN-1, SN-2, SN-3, SN-4, SN-5 y Diosgenona) se muestran en la Tabla 4. Los valores obtenidos presentan una correlación estadísticamente significativa ( $p$-valor $=0,001$ ), con un nivel de confianza del 95\%, los coeficientes de correlación para los modelos lineales fueron de 0,999; indicando buena proporcionalidad entre la concentración y el área (López, et al. 2014).

\section{TABLA 3. EXTRACTOS OBTENIDOS DE LA PLANTA S. nudum}

\begin{tabular}{|c|c|c|c|c|}
\hline Municipio & Vereda & Fecha de colecta & Tipo de extracto & Porcentaje de extracto $(\mathrm{m} / \mathrm{m})$ \\
\hline \multirow{6}{*}{ Cocorná } & \multirow{6}{*}{ La piñuela } & \multirow{3}{*}{ Nov-2011 } & Hexano & 1,19 \\
\hline & & & Diclorometano & 5,21 \\
\hline & & & Metanol & 31,37 \\
\hline & & \multirow{3}{*}{ Jun-2011 } & Hexano & 1,70 \\
\hline & & & Diclorometano & 4,81 \\
\hline & & & Metanol & 28,54 \\
\hline \multirow{6}{*}{ Tumaco } & \multirow{6}{*}{ Inguapí el Guadual } & \multirow{3}{*}{ Nov-2010 } & Hexano & 1,52 \\
\hline & & & Diclorometano & 4,71 \\
\hline & & & Metanol & 23,10 \\
\hline & & \multirow{3}{*}{ Jun-2011 } & Hexano & 2,41 \\
\hline & & & Diclorometano & 6,06 \\
\hline & & & Metanol & 19,84 \\
\hline
\end{tabular}

TABLA 4. PARÁMETROS CROMATOGRÁFICOS PARA LA CUANTIFICACIÓN DE ESTEROIDES DE S. nudum

\begin{tabular}{c|c|c|c|c|c|c} 
Compuesto & $\begin{array}{c}\text { Rango Lineal } \\
\mathbf{( \mu g / m L )}\end{array}$ & Ecuación & $\mathbf{r}^{\mathbf{2}}$ & $\begin{array}{c}\text { LD } \\
(\boldsymbol{\mu g} / \mathbf{m L})\end{array}$ & $\begin{array}{c}\text { LC } \\
(\boldsymbol{\mu g} / \mathbf{m L})\end{array}$ & $\begin{array}{c}\text { TR } \\
(\mathbf{m i n})\end{array}$ \\
\hline $\mathbf{S N}-\mathbf{1}$ & $0,5-160$ & $\mathrm{~A}=24,7^{*} \mathrm{C}$ & 0,999 & 0,10 & 0,33 & 17,657 \\
\hline $\mathbf{S N - 2}$ & $0,5-160$ & $\mathrm{~A}=27,4^{*} \mathrm{C}$ & 0,999 & 0,09 & 0,30 & 16,568 \\
\hline $\mathbf{S N}-3$ & $0,5-160$ & $\mathrm{~A}=16,8^{*} \mathrm{C}$ & 0,999 & 0,14 & 0,46 & 14,850 \\
\hline $\mathbf{S N}-4$ & $3,0-160$ & $\mathrm{~A}=15,7^{*} \mathrm{C}$ & 0,999 & 0,75 & 2,50 & 12,357 \\
\hline SN-5 & $0,5-160$ & $\mathrm{~A}=20,5^{*} \mathrm{C}$ & 0,999 & 0,15 & 0,50 & 14,145 \\
\hline Diosgenona & $3,0-160$ & $\mathrm{~A}=21,5^{*} \mathrm{C}$ & 0,999 & 0,82 & 2,73 & 37,492
\end{tabular}

A: área, C: concentración, $\mathbf{r}^{2}$ : coeficiente de determinación, LD: límite de detección, LC: límite de cuantificación y TR: tiempo de retención. 
En la Figura 1 se muestra el cromatograma de los patrones a una concentración de $120 \mu \mathrm{g} / \mathrm{mL}$, donde se observa una definición y resolución de los picos sin problemas de coelución entre ellos.

Efecto de la región en la composición de esteroides en Solanum nudum

En la Tabla 5 Se evidencia que en los extractos obtenidos con solvente hexano hay presencia de los esteroides de polaridad media (SN-1 y SN-2) y polaridad baja (Diosgenona). Los compuestos de polaridad alta como SN-4, SN-5 y SN3, no fueron detectados. Para los extractos obtenidos con diclorometano y metanol de detectaron todos los esteroides de interés, predominando los compuestos de polaridad media (SN-1 y SN-2) en diclorometano y los compuestos de polaridad alta (SN-4, SN-5 y SN3) en metanol.

El contenido de los compuestos esteriodales en los tres extractos de los dos municipios, conserva la tendencia, respecto al efecto de la polaridad del solvente en la extracción, difiere significativamente en la cantidad de los compuestos, es decir SN-1, SN-2, SN-3 y SN-4 son menores en Tumaco que en Cocorná, pero SN-5 y Diosgenona son mayores en Tumaco.

Las diferencias en la cantidad de compuestos esteroidales entre las dos regiones estudiadas puede ser una consecuencia de la interacción de las plantas con su respectivo entorno biótico (herbívoros y polinizadores) y abiótico (composición química del suelo y microclima). La diferencia en el contenido esteroidal entre los extractos de Cocorná y Tumaco puede explicarse por las diferencias encontradas en algunos aspectos ecológicos de las plantas en su respectivo ambiente. Previamente se había reportado que las plantas de S. nudum en Cocorná presentaron una mayor cantidad de especies de visitantes florales y frutos/mes. Además, se encontró una menor prevalencia de insectos (hervíboros, parásitos y huéspedes temporales) en las plantas de Cocorná comparado con las provenientes de Tumaco (Tabla 1).

\section{Figura 1. Cromatograma de la mezcla de patrones: SN-1, SN-2, SN-3, SN-4, SN-5, Diosgenona}

\section{Fuente: autores}

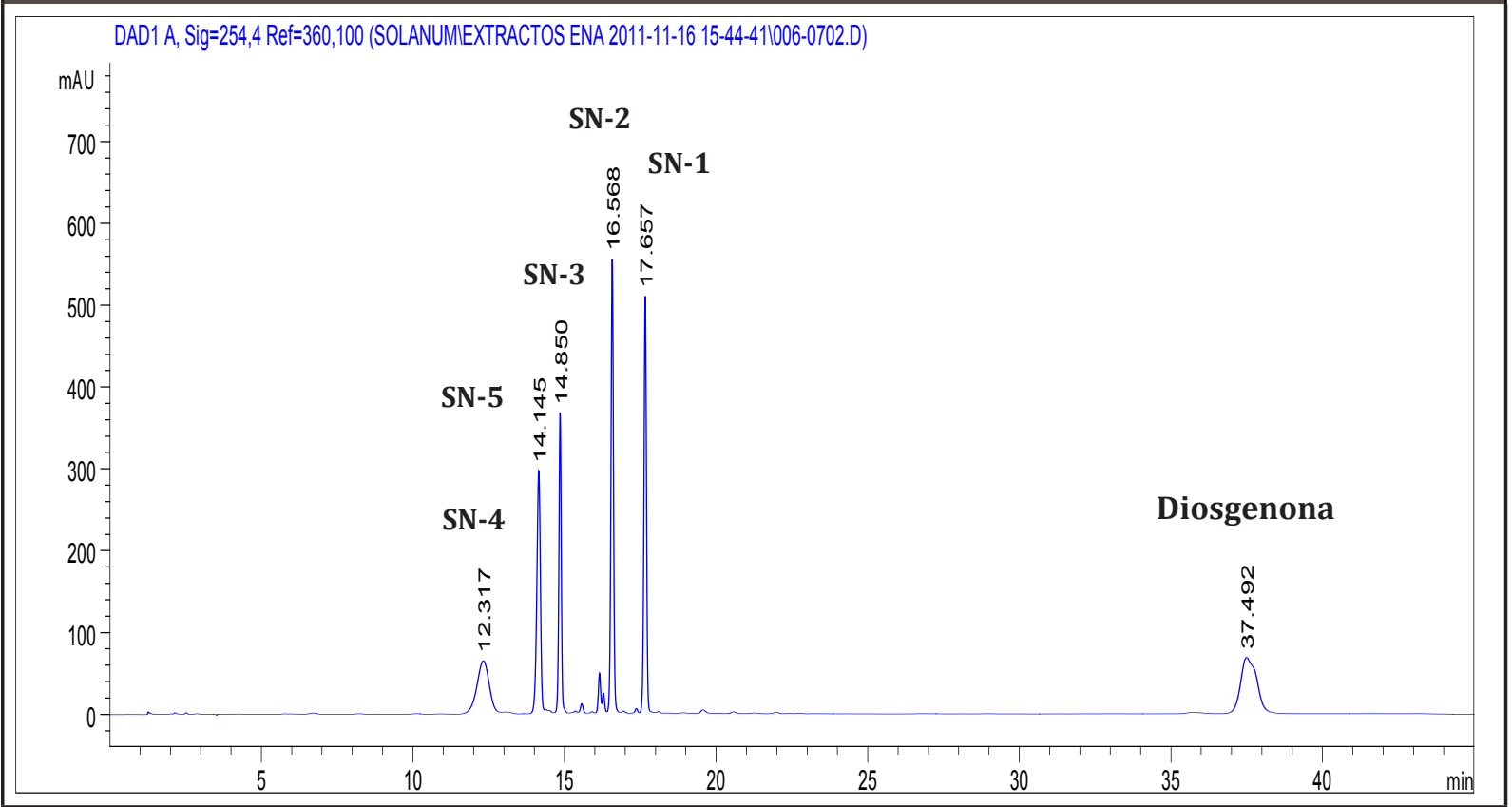


TABLA 5. CONTENIDO DE COMPUESTOS ESTEROIDALES EN EXTRACTOS DE S. nudum $(\mu \mathrm{g} / \mathrm{mg}$ ) DE LAS COLECTAS REALIZADAS EN COCORNÁ Y TUMACO EN EL MES DE NOVIEMBRE DE 2010

\begin{tabular}{|c|c|c|c|}
\hline Extracto & Compuesto & Cocorná & Tumaco \\
\hline \multirow{6}{*}{ Hexano } & SN-1 & $35,7 \pm 0,2$ & $3,2 \pm 0,2$ \\
\hline & $\mathrm{SN}-2$ & $22,5 \pm 0,4$ & $6,2 \pm 0,3$ \\
\hline & SN-3 & ND & ND \\
\hline & $\mathrm{SN}-4$ & ND & ND \\
\hline & SN-5 & ND & ND \\
\hline & Diosgenona & $85 \pm 1$ & $111 \pm 3$ \\
\hline \multirow{6}{*}{ Diclorometano } & SN-1 & $96 \pm 1$ & $9,7 \pm 0,3$ \\
\hline & SN-2 & $191 \pm 11$ & $85 \pm 3$ \\
\hline & SN-3 & $98,6 \pm 0,2$ & $17,1 \pm 0,5$ \\
\hline & $\mathrm{SN}-4$ & $89 \pm 2$ & $64 \pm 3$ \\
\hline & SN-5 & $9,7 \pm 0,5$ & $38 \pm 1$ \\
\hline & Diosgenona & $12,7 \pm 0,4$ & $55 \pm 2$ \\
\hline \multirow{6}{*}{ Metanol } & SN-1 & $22 \pm 1$ & $1,63 \pm 0,07$ \\
\hline & $\mathrm{SN}-2$ & $33 \pm 2$ & $9,6 \pm 0,6$ \\
\hline & SN-3 & $107,2 \pm 0,5$ & $23,3 \pm 0,7$ \\
\hline & SN-4 & $114,1 \pm 0,7$ & $71 \pm 2$ \\
\hline & SN-5 & $16 \pm 1$ & $56,5 \pm 0,9$ \\
\hline & Diosgenona & $5,5 \pm 0,3$ & $12,3 \pm 0,9$ \\
\hline
\end{tabular}

Para el caso de compuestos químicos tipo esteroides se ha reportado que su pico máximo de biosíntesis se da en la floración de las plantas y que estos pueden ser tóxicos para microorganismos, insectos y otros animales, actuando como defensa contra depredadores, parásitos y herbívoros (Díaz, 2009), pero estos compuestos también pueden actuar como sustancias atrayentes de polinizadores, favoreciendo de este modo las ventajas competitivas de la planta frente a otras en cuanto a la mayor probabilidad de generación de frutos (Díaz, 2009). De esta manera, la producción de metabolitos secundarios influye indirectamente sobre la sobrevivencia de la planta y la dinámica ecológica de esta planta en su ecosistema (Granados, et al. 2008; Wink, 2003). Sin embargo, la supervivencia de las plantas bajo condiciones de ataque depende de la capacidad de las mismas de percibir el estímulo, generar y transmitir las señales correspondientes y así iniciar los cambios fisiológicos y químicos necesarios para la biosíntesis de metabolitos (Zobayed, et al. 2007).

En este sentido, se ha reportado que los metabolitos que actúan como defensa contra los depredadores de las plantas reducen el rendimiento, la supervivencia y la reproducción de estos atacantes $\mathrm{y}$, por tanto, se espera que las plantas que tengan la capacidad de producir mayor cantidad de estos metabolitos sean menos atacadas por este tipo de plagas (Steppuhn, et al. 2004; Kamel y Souad, 2009).

Respecto a la luminosidad, las plantas de Cocorná tienen una mayor disponibilidad de luz en su entorno, lo que permite que los procesos fotosintéticos se lleven a cabo de manera favorable y consecuentemente aporten, en mayor medida, al crecimiento y desarrollo de la planta y a la producción de sus metabolitos (Briskin yGawienowski, 2001; Mosaleeyanon, et al. 2005) si se compara con las plantas de Tumaco (Tabla 1).

Para los compuestos SN-1, SN-2, SN-3, SN-4, SN-5 y la Diosgenona aislados inicialmente de la planta $S$. nudum colectada en Tumaco se ha planteado una ruta biosintética (Saez, et al. 1998), como se ilustra en la Figura 2, donde el compuesto SN-2 es considerado un metabolito sustrato, SN-1, SN-3 y SN-4 metabolitos intermediarios, SN-5 metabolito sustrato e intermediario y finalmente la Diosgenona como metabolito producto.

En la Figura 3 se ilustra una posible ruta biosintética del metabolismo de los compuestos esteroidales de la planta de $S$. nudum colectada en Cocorná, donde el compuesto $\mathrm{SN}-2$ es considerado un metabolito sustrato, $\mathrm{SN}-1, \mathrm{SN}-5$ y SN-4 metabolitos intermediarios, SN-3 y Diosgenona son metabolitos producto. 
Figura 2. Ruta biosintética de esteroides de S. nudum proveniente de Tumaco.

Fuente: autores

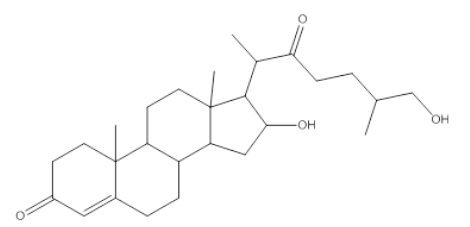

Tumacona B (SN-2)

I Glucosidación

$$
\text { I }
$$

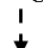

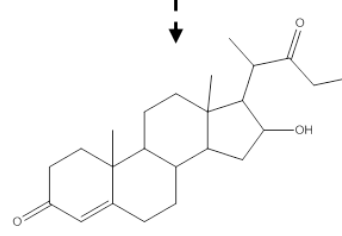

Tumacosido B (SN-5)

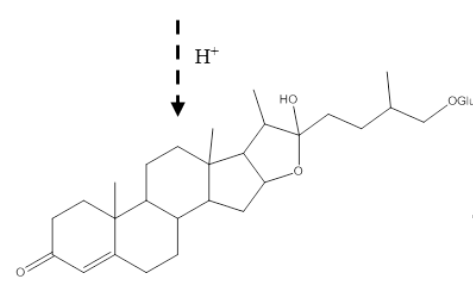

Tumaquenona (SN-4)

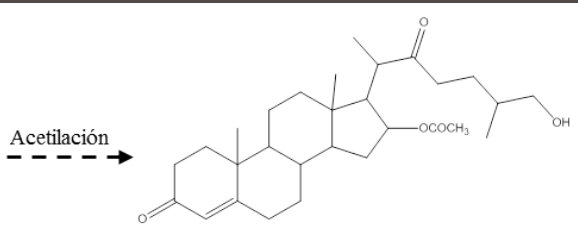

Tumacona A (SN-1)

I

I Glucosidación

I

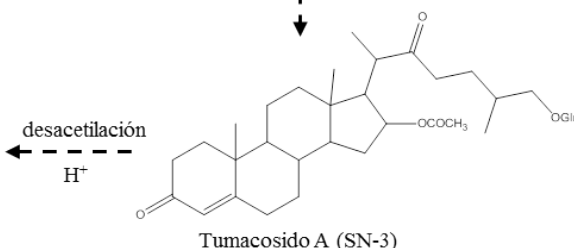

Tumacosido A (SN-3)

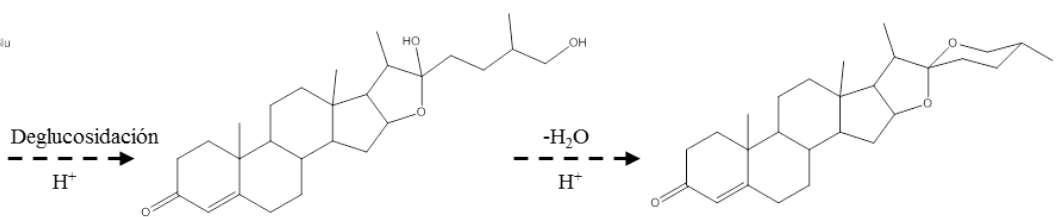

Diosgenona

Figura 3. Posible ruta biosintética de esteroides de S. nudum proveniente de Cocorná

Fuente: autores

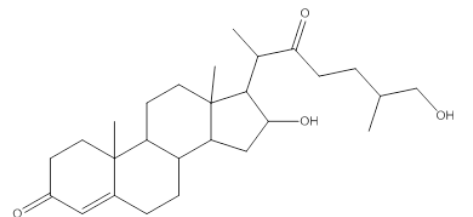

Tumacona B (SN-2)

I Glucosidación

$\downarrow$

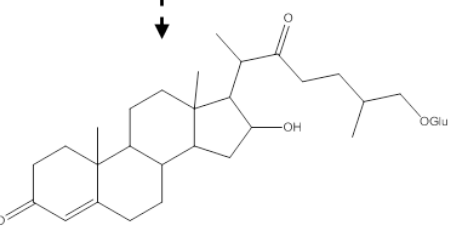

Tumacosido B (SN-5)

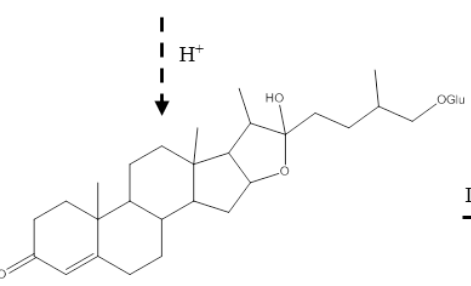

Tumaquenona (SN-4)

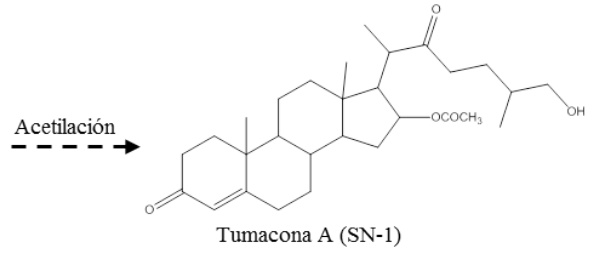

I Glucosidación

I

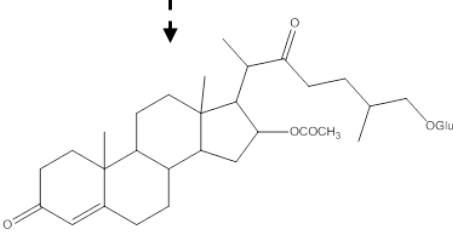

Tumacosido A (SN-3)

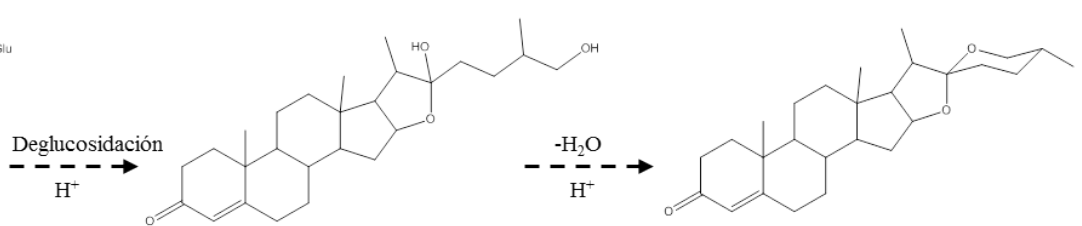

Diosgenona 
Efecto del mes de recolección de $S$. nudum en la composición de esteroides

En los meses de noviembre de 2010 y junio de 2011 correspondientes a las colectas del material vegetal coincidieron con la presencia del fenómeno de La Niña para algunas regiones de Colombia; "La Niña" se manifiesta principalmente por un enfriamiento de las aguas del Océano Pacífico Tropical central y oriental frente a las costas del Perú, Ecuador y sur de Colombia, "La Niña" favorece el incremento de las lluvias en gran parte del país en particular sobre las regiones Caribe y Andina (IDEAM, 2010). Monitoreos realizados por el IDEAM, indicaron que en noviembre de 2010 se evidenció la fase de maduración del fenómeno de La Niña, y se registraron lluvias moderadamente por encima del promedio para la zona de Cocorná, mientras que para la zona de Tumaco se registraron un régimen de lluvias moderadamente por debajo de lo normal. Según el IDEAM, el mes de junio de 2011, correspondió a la finalización de la primera temporada de lluvia. Sin embargo, para la zona de Cocorná se registraron lluvias ligeramente por encima de lo normal, mientras que para Tumaco se observó lo contrario (IDEAM, 2011).

En la Tabla 6 se puede apreciar el contenido de esteroides para las colectas de $S$. nudum realizada en el mes de noviembre del 2010 y junio del 2011 en los municipios de Cocorná y Tumaco.

TABLA 6. CONTENIDO DE ESTEROIDES EN EXTRACTOS DE S. nudum EN LOS MUNICIPIOS DE COCORNÁ Y TUMACO ( $\mu \mathrm{g} /$ mg DE EXTRACTO)

\begin{tabular}{|c|c|c|c|c|c|}
\hline \multirow{2}{*}{ Extracto } & \multirow{2}{*}{ Compuesto } & \multicolumn{2}{|c|}{ Cocorná } & \multicolumn{2}{|c|}{ Tumaco } \\
\hline & & Nov-2010 & Jun-2011 & Nov-2010 & Jun-2011 \\
\hline \multirow{6}{*}{ Hexano } & $\mathrm{SN}-1$ & $35,7 \pm 0,2$ & $5,2 \pm 0,3$ & $3,2 \pm 0,2$ & $10,2 \pm 0,8$ \\
\hline & $\mathrm{SN}-2$ & $22,5 \pm 0,4$ & $3,4 \pm 0,3$ & $6,2 \pm 0,3$ & $14 \pm 1$ \\
\hline & SN-3 & ND & ND & ND & ND \\
\hline & $\mathrm{SN}-4$ & ND & ND & ND & ND \\
\hline & SN-5 & ND & ND & ND & ND \\
\hline & Diosgenona & $85 \pm 1$ & $12 \pm 1$ & $111 \pm 3$ & $179 \pm 2$ \\
\hline \multirow{6}{*}{ Diclorometano } & SN-1 & $96 \pm 1$ & $9,4 \pm 0,1$ & $9,7 \pm 0,3$ & $22 \pm 1$ \\
\hline & $\mathrm{SN}-2$ & $191 \pm 11$ & $105,4 \pm 0,6$ & $85 \pm 3$ & $115 \pm 3$ \\
\hline & SN-3 & $98,6 \pm 0,2$ & $97 \pm 2$ & $17,1 \pm 0,5$ & $10,9 \pm 0.9$ \\
\hline & SN-4 & $89 \pm 2$ & $95 \pm 2$ & $64 \pm 3$ & $47 \pm 3$ \\
\hline & SN-5 & $9,7 \pm 0,5$ & $18,6 \pm 0,9$ & $38 \pm 1$ & $28 \pm 3$ \\
\hline & Diosgenona & $12,7 \pm 0,4$ & $3,9 \pm 0,2$ & $55 \pm 2$ & $61,9 \pm 0,7$ \\
\hline \multirow{6}{*}{ Metanol } & SN-1 & $22 \pm 1$ & $1,51 \pm 0,02$ & $1,63 \pm 0,07$ & $5,9 \pm 0,4$ \\
\hline & $\mathrm{SN}-2$ & $33 \pm 2$ & $3,19 \pm 0,08$ & $9,6 \pm 0,6$ & $29 \pm 3$ \\
\hline & SN-3 & $107,2 \pm 0,5$ & $66 \pm 4$ & $23,3 \pm 0,7$ & $13 \pm 3$ \\
\hline & $\mathrm{SN}-4$ & $114,1 \pm 0,7$ & $147 \pm 2$ & $71 \pm 2$ & $46 \pm 2$ \\
\hline & SN-5 & $16 \pm 1$ & $24,3 \pm 0,7$ & $56,5 \pm 0,9$ & $44 \pm 2$ \\
\hline & Diosgenona & $5,5 \pm 0,3$ & $1,01 \pm 0,08$ & $12,3 \pm 0,9$ & $25 \pm 2$ \\
\hline
\end{tabular}


El mayor contenido de metabolitos en los extractos de $S$. nudum fue encontrado en noviembre para el municipio de Cocorná y en junio para el municipio de Tumaco. Posiblemente el cambio fisiológico inducido por el estrés provocado por las variaciones de temperatura y precipitaciones asociadas al fenómeno de La Niña, promueve producción de metabolitos secundarios probablemente para la restauración de mecanismo de defensa. Así también, la tasa de transpiración de la hoja está significativamente influenciada por la temperatura contribuyendo al aumento en las concentraciones de los compuestos químicos de la planta (Zabayed, et al. 2005).

Respecto al municipio de Cocorná para los extractos de la colecta del mes de noviembre, los compuestos esteroidales como Diosgenona es mayoritario en el extracto de hexano, $\mathrm{SN}-1$ y SN-2 en el extracto de diclorometano y SN-3, SN-4 y SN-5 en el extracto de metanol. Sin embargo, entre los meses de colecta los compuestos SN-4 y SN-5 fueron mayores para el mes de junio en los extractos de diclorometano y metanol.

Para los extractos de la colecta del mes de junio del municipio de Tumaco, los compuestos esteroidales como Diosgenona fue mayoritario hexano, $\mathrm{SN}-1$ y SN-2 en el extracto de diclorometano y SN-3, SN-4 y SN-5 en el extracto de metanol. Sin embargo, entre los meses de colecta los compuestos $\mathrm{SN}-4$ y SN-5 fueron mayores para el mes de noviembre en los extractos de diclorometano y metanol.

\section{Actividad antiplasmodial de los ex-} tractos obtenidos de la planta $S$. nudum

En la Tabla 7 se presentan la actividad antiplasmodial $\left(\mathrm{IC}_{50}<50 \mu \mathrm{g} / \mathrm{mL}\right)$ de los extractos de la planta $S$. nudum colectada en Cocorná y Tumaco.

Se observan diferencias estadísticamente significativas en la actividad antiplasmodial $(\mathrm{p}<0,05)$, entre los meses de colecta. Para Cocorná, los extractos con mayor efecto antiplasmodial $\left(\mathrm{IC}_{50}\right.$ más baja) fueron los de hexano (IC 50 Hexano $24 \pm 4 \mu \mathrm{g}$ / $\mathrm{mL}$ ) y diclorometano ( $\mathrm{IC}_{50 \text { Diclorometano }} 23 \pm 2 \mu \mathrm{g} / \mathrm{mL}$ ) de la colecta noviembre, los cuales no presentaron diferencia estadísticamente significativa $(p<0,05)$, atribuyendo a los compuestos $\mathrm{SN}-1$ y Diosgenona la actividad antiplasmodial, dado que comparando las actividades entre los extractos de diclorometano en los dos meses de colecta, para los cuales el extracto de noviembre que es 10 veces más activo, no se presentó diferencia estadísticamente significativa entre los componentes SN-3 y SN-4 con el extracto de la colecta de junio.

Respecto a Tumaco se presentó mayor efecto antiplasmodial en los extractos de la colecta de junio ( IC $_{50 \text { Hexano }}: 17 \pm 1 \mu \mathrm{g} / \mathrm{mL}$; IC 50 Diclorometano $21 \pm 1 \mu \mathrm{g}$ / $\mathrm{mL} ; \mathrm{IC}_{50 \text { Metanol }} 30 \pm 2 \mu \mathrm{g} / \mathrm{mL}$ ), para los cuales se tiene un mayor contenido de compuestos SN-1 y diosgenona, principalmente Diosgenona la cual se presenta en mayor cantidad (Tabla 6).

TABLA 7. ACTIVIDAD ANTIPLASMÓDICA DE LOS EXTRACTOS $(\mu \mathrm{g} / \mathrm{mL})$ DE S. nudum COLECTADA EN COCORNÁ Y TUMACO

\begin{tabular}{|c|c|c|c|c|c|c|}
\hline \multicolumn{4}{|c|}{ TUMACO } & \multicolumn{3}{|c|}{ COCORNÁ } \\
\hline \multirow[t]{2}{*}{ Extractos } & Noviembre & Junio & P valor* & Noviembre & Junio & P valor* \\
\hline & Me $\pm \mathrm{DS}$ & Me $\pm \mathrm{DS}$ & & Me $\pm \mathrm{DS}$ & Me $\pm \mathrm{DS}$ & \\
\hline Hexano & $40,44 \pm 0,71$ & $17,46 \pm 1,35$ & 0,0001 & $23,99 \pm 3,69$ & $100,00 \pm 0,00$ & $<0,0001$ \\
\hline Diclorometano & $39,06 \pm 4,12$ & $21,22 \pm 1,30$ & 0,001 & $23,43 \pm 1,56$ & $88,00 \pm 20,79$ & 0,032 \\
\hline Metanol & $47,56 \pm 7,45$ & $29,74 \pm 1,50$ & 0,022 & $50,29 \pm 1,60$ & $64,79 \pm 13,50$ & 0,203 \\
\hline \multicolumn{7}{|c|}{$\begin{array}{l}\text { * Prueba t-student } p<0.05 \text {, se compararon las concentracione } \\
\text { es de noviembre y junio en los municipios de Tumaco y Cocorná. }\end{array}$} \\
\hline \multicolumn{7}{|c|}{ Me (promedio) y DS (desviación estándar) } \\
\hline
\end{tabular}


Una mejor actividad antiplasmodica en Cocorná y Tumaco se observó en los periodos de lluvia de cada localidad, cuando se presentó la mayor concentración de los compuestos SN-1 y Diosgenona (Tabla 8), a los cuales se les atribuye la actividad antiplasmodial (Pabón, et al. 2002; López, et al. 2009).

La actividad antiplasmodial del extracto de diclorometano de Cocorná colecta noviembre $\left(\mathrm{IC}_{50}\right.$ $23 \pm 2 \mu \mathrm{g} / \mathrm{mL}$ ) y el extracto de diclorometano de Tumaco colecta junio ( IC $_{50 \text { Diclorometano }} 21 \pm 1$ $\mu \mathrm{g} / \mathrm{mL}$ ) no presentaron diferencias estadísticamente significativa ( $\mathrm{p}$-valor $=0,001)$, teniendo en cuenta que SN-1 fue mayor para Cocorná y Diosgenona para Tumaco (Tabla 8).

\section{Actividad citotóxica de los extractos} obtenidos de la planta $S$. nudum

En la Tabla 9 se presentan la actividad citotóxica de los extractos de la planta $S$. nudum. Se obser- va diferencias estadísticamente significativas ( $\mathrm{p}<$ $0,05)$, tanto en las localidades como entre los meses de colecta.

* Prueba t-student $\mathrm{p}<0,05$; se compararon las concentraciones tóxicas $50\left(\mathrm{TC}_{50}\right)$ de cada extracto entre los meses de noviembre y junio en los municipios de Tumaco y Cocorná.

\section{Me (promedio) y DS (desviación estándar)}

Los extractos con mayor efecto citotóxico (TC $_{50}$ $<100 \mathrm{ug} / \mathrm{mL}$ ), fueron los obtenidos con hexano y diclorometano provenientes de la localidad de Tumaco colecta del mes de junio. Para Cocorná los extractos con mayor efecto citotóxico fueron los obtenidos con hexano y diclorometano de la colecta noviembre 2010. Aunque los extractos de Cocorná de la colecta de junio 2011 no presentaron actividad cititóxica $\left(\mathrm{TC}_{50}>100 \mathrm{ug} / \mathrm{mL}\right)$, estos no tienen actividad antiplasmodial.

TABLA 8. ACTIVIDAD ANTIPLASMÓDICA ( $\mu \mathrm{g} / \mathrm{mL})$ DE LOS EXTRACTOS DE S. nudum COLECTADA EN COCORNÁ Y TUMACO.

\begin{tabular}{|c|c|c|c|c|c|}
\hline Extracto & Compuesto & $\begin{array}{c}\text { Cocorná } \\
\text { Nov-2010 }\end{array}$ & $\begin{array}{c}\text { Actividad } \\
\text { antiplasmódica } \\
(\mu \mathrm{g} / \mathrm{mL})\end{array}$ & $\begin{array}{l}\text { Tumaco } \\
\text { Jun-2011 }\end{array}$ & $\begin{array}{c}\text { Actividad } \\
\text { antiplasmódica } \\
(\mu \mathrm{g} / \mathrm{mL})\end{array}$ \\
\hline \multirow{6}{*}{ 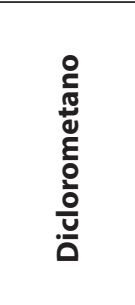 } & $\mathrm{SN}-1$ & $96 \pm 1$ & \multirow{6}{*}{$23 \pm 2$} & $22 \pm 1$ & \multirow{6}{*}{$21 \pm 1$} \\
\hline & SN-2 & $191 \pm 11$ & & $115 \pm 3$ & \\
\hline & $\mathrm{SN}-3$ & $98,6 \pm 0,2$ & & $10,9 \pm 0.9$ & \\
\hline & SN-4 & $89 \pm 2$ & & $47 \pm 3$ & \\
\hline & SN-5 & $9,7 \pm 0,5$ & & $28 \pm 3$ & \\
\hline & Diosgenona & $12,7 \pm 0,4$ & & $61,9 \pm 0,7$ & \\
\hline
\end{tabular}

TABLA 9. CONCENTRACIÓN CITOTÓXICA $50\left(C_{50}\right)$ DE LOS EXTRACTOS DE S. nudum COLECTADA EN TUMACO Y COCORNÁ EN EL MES DE NOVIEMBRE Y JUNIO.

\begin{tabular}{|l|c|c|c|c|c|c|}
\hline \multicolumn{3}{|c|}{ TUMACO } & \multicolumn{3}{c|}{ COCORNÁ } \\
\hline Extractos & NOVIEMBRE & JUNIO & P VALOR* & NOVIEMBRE & JUNIO & P VALOR * \\
\hline Mexano & Me \pm DS & Me $\pm D S$ & & Me $\pm D S$ & Me $\pm D S$ & \\
\hline Diclorometano & $36,46 \pm 8,92$ & $19,87 \pm 0,81$ & 0,028 & $61,31 \pm 0,77$ & $200,00 \pm 0,00$ & $<0,0001$ \\
\hline Metanol & $126,43 \pm 21,92$ & $92,65 \pm 5,65$ & 0,051 & $84,55 \pm 8,71$ & $111,37 \pm 17,79$ & 0,054
\end{tabular}




\section{Conclusiones}

La diferencia en la composición de los extractos obtenidos de de $S$. nudum se debe a las diferencias en algunos aspectos ecológicos de las plantas en su respectivo ambiente. A demás el contenido de compuestos esteroidales y la actividad antiplasmodial fue superior para los extractos de las regiones Cocorná y Tumaco en los meses de noviembre 2010 y junio 2011 donde la precipitación promedio del lugar fue alterada debido al fenómeno de La Niña el cual promovió la producción de los metabolitos esteroidales.

El incremento de las lluvias en los municipios de Cocorná y Tumaco favorecieron la productividad de los componentes SN-1, SN-2, SN-3 y Diosgenona, sin embargo, es importante resaltar que en el periodo donde no estuvo presente el fenómeno de la Niña, los compuestos SN-4 y SN-5 fueron los mayoritarios

Aunque se ha reportado que los esteroides aislados de $S$. nudum no presentan actividad tóxica, posiblemente la toxicidad encontrada en los extractos que presentaron mayor actividad antiplasmodial puede deberse a componente diferentes a los compuestos estereoidales trabajados en este estudio. Los extractos obtenidos con metanol tanto para Cocorná y Tumaco en los meses de noviembre 2010 y junio 2011, presentaron menor actividad antiplasmodial, comparados con los otros extractos, pero fueron menos citotóxicos.

\section{Referencias}

Alvarez, G; Pabón, A; Carmona, J; Blair, S. (2004). Evaluation of clastogenic potential of the antimalarial plant Solanum nudum. Phytother Res, 18(10) octubre, pp. 845-848.

Blair, S; Madrigal, B. Plantas antimaláricas de Tumaco: Costa Pacífica Colombiana, in: E.U.d. Antioquia (Ed.), Medellín, 2005, pp. 266-270.

Boira, H; Blanquer, A. (1998). Environmental factors affecting chemical variability of essential oils in Thymus piperella L. Biochem Syst Ecol. 26 diciembre, pp. 811-822.
Bravo, B; Sauvain, M; Gimenez, T; Muñoz, 0; Callapa, J; Le Men, O; Massiot, G; Lavaud, C. (1999). Bioactive phenolic glycosides from Amburana cearensis. Phytochemistry. 50 enero, pp. 71-74.

Briskin, D.P.; Gawienowski, M.C. (2001). Differential effects of light and nitrogen on production of hypericins and leaf glands in Hypericum perforatum. Plant Physiol. Bioch, 39 dicienbre, pp. 1075-1081.

Celiktas, O; Kocabas, E; Bedir, E; Sukan, F; Ozek, T; Baser, K. (2007) Antimicrobial activities of methanol extracts and essential oils of Rosmarinus officinalis, depending on location and seasonal variations, Food Chem. 100, pp. 553-559.

Christos N. Hassiotis, Diamanto M. Lazari. (2010). Decomposition process in the Mediterranean region. Chemical compounds and essential oil degradation from Myrtus communis. International Biodeterioration \& Biodegradation, 64 junio, pp. 356-362.

Desjardins, R; Canfield, C; Haynes, D; Chulay, J. (1979). Quantitative assessment of antimalarial activity In Vitro by a semiautomated microdilution technique. Antimicrob Agents Chemother, 16 diciembre, pp. 710-718.

Diaz, L. (2009). Interacciones moleculares entre plantas y microorganismos: saponinas como defensas químicas de las plantas y su tolerancia a los microorganismos, Estudios Transdisciplinarios. 1 junio, pp. 32-55.

Echeverri, M; Blair, S; Carmona, J; Perez, P. (2001). Effect of Solanum nudum extracts on the liver of mice infected with Plasmodium berghei. Am J Chin Med. 29 septiembre, pp. 477-484.

Granados, D; Ruiz, P; Barrera H. (2008). Ecologia de la herbivoria, Chapingo. 14 enero, pp. 51-64.

IDEAM, Boletín informativo sobre el monitoreo de los fenómenos de "El Niño" y “La Niña”, in, 2010.

IDEAM, Boletín informativo sobre el monitoreo de los fenómenos de "El Niño" y “La Niña”, in, 2011.

Kamel, A; Souad, E. (2009). Is there a relationship between the level of plant metabolites in Cucumber and Globe Cucumber and the degree of Insect Infestation?, Not. Bot. Horti Agrobo. 37, pp. 144-156.

Londoño, B; Arango, E; Zapata, C; Herrera, S; Saez, J; Blair, S; Carmona, J. (2006). Effect of Solanum nudum Dunal (Solanaceae) steroids on hepatic trophozoites of Plasmodium vivax. Phytother Res, 20, abril, pp. 267273. 
López, E; Barbosa, C; Mesa, A; Araque., P. (2011). Actividad antiplasmodial de extractos etanólicos Solanum nudum dunal: efecto en el proceso descomposición del material vegetal.

Revista Vitae, 18(2) octubre, pp. S313-S314.

López, E; López, C; Araque, P; Blair, S; Pabón, A. (2014). Simultaneous quantification of antimalarial steroids in solanum nudum extracts by High Performance Liquid Chromatography (HPLC). Revista Politecnica, 10(18) enero, pp 23-33.

López, M.L.; Blair, S; Saez, J; Segura, C. (2009). Effect of Solanum nudum steroids on uninfected and Plasmodium falciparum-infected erythrocytes. Mem. Inst. Oswaldo Cruz. 104, pp. 683-688.

Mosaleeyanon, K; Zobayed, S; Afreen, F; Kozai, T. (2005). Relationships between net photosynthetic rate and secondary metabolite contents in St. John's wort. Plant Sci, 169 septiembre, pp. 523-531.

Mosmann, T. (1983). Rapid colorimetric assay for cellular growth and survival: application to proliferation and cytotoxicity assays. Journal of Immunological Methods, 65 diciembre, pp. 55-63.

Morales, P. Aspectos ecológicos de Solanum nudum (Dunal 1816), una planta promisoria contra la malaria, trabajo de pregrado en Biología, Universidad de Antioquia, Medellín, 2011, pp. 1-85.

Orozco, C.I.; Alba, A; Beltrán, G; Orejuela, A; Sarmiento, Y; Vélez, J.M. Solanum nudum Dunal En Bernal, R., S.R. Gradstein \& M. Celis (eds.). 2015. Catálogo de plantas y líquenes de Colombia. Instituto de Ciencias Naturales, Universidad Nacional de Colombia, Bogotá.

Pabón, A; Carmona, J; Maestre, A; Camargo, M; Blair, S. (2002). Inhibition of $P$. falciparum by steroids isolated from Solanum nudum. Phytother Res, 16 febrero, pp. 59-62.

Pabón, A; Blair, S; Carmona, J; Zuleta, M; Saez, J. (2003). Evaluation of the mutagenicity of antimalarial products isolated from Solanum nudum (Solanaceae). Pharmazie. 58(4) abril, pp. 263-267.

Reed, G; Lynn, F; Meade, B. (2002). Use of coefficient of variation in assessing variability of quantitative assays. Clin Diagn Lab Immunol. 9 noviembre, pp.1235-1239.

Saez, J; Cardona, W; Espinal, D; Blair, S; Mesa, J; Bocar, M; Jossang, A. (1998). Five new steroids from Solanum nudum, Tetrahedron, 54 septiembre, pp. 1077110778.
Stafford, G.I.; Jäger, A.K.; van Staden. J. (2005). Effect of storage on the chemical composition and biological activity of several popular South African medicinal plants. Ethnopharmacology. 97 febrero, pp. 107115.

Steppuhn, A, Gase, K; Krock, B; Halitschke, R; Baldwin, I. (2004). Nicotine's defensive function in nature. Plos Biol, 2(8) agosto, pp.1074 -1080.

Walliker, D; Quakyi, I.A; Wellems, T. E; Mccutchan, T. F; Szarfman, A; London, W. T; Carter, R. (1987). Genetic Analysis of the Human Malaria Parasite Plasmodium falciparum. American Association for the Advancement of Science Stable, 236(4809), 1661-1666.

Wink, W. (2003). Evolution of secondary metabolites from an ecological and molecular phylogenetic perspective. Phytochemistry, 64 septiembre, pp. 3-19.

Zobayed, S; Afreen, F; Kozai, T. (2005). Temperature stress can alter the photosynthetic efficiency and secondary metabolite concentrations in St. John's wort. Plant Physiol. Bioch, 43 octubre, pp. 977-984.

Zobayed, S; Afreen, F; Kozai, T. (2007). Phytochemical and physiological changes in the leaves of St. John's wort plants under a water stress condition. Environ Exp Bot. 59 marzo, pp. 109-116.

\section{PARA CITAR ESTE ARTÍCULO / TO REFERENCE THIS ARTICLE / PARA CITAR ESTE ARTIGO /}

López-Barrios, E.P.; Pabón-Vidal, A.L; Blair-Trujillo, S.; Morales-Morales, P.A.; Peláez-Jaramillo, C.A.; Araque-Marín, P. (2018). Actividad antiplasmodial in vitro de metabolitos secundarios de Solanum nudum provenientes de dos regiones de Colombia. Revista EIA, 15(30), julio-diciembre, pp. 25-39. [Online]. Disponible en: https://doi.org/10.24050/reia. v15i30.1033 